\title{
Pattern Competition in the Photorefractive Semiconductors
}

\author{
Yuo-Hsien Shiau ${ }^{1, *}$ and Chin-Kun $\mathrm{Hu}^{2,3,+}$ \\ ${ }^{1}$ National Center for Theoretical Sciences (Physics Division) 101, Section 2 Kuang Fu Road, \\ Hsinchu, Taiwan 300, ROC \\ ${ }^{2}$ Institute of Physics, Academia Sinica, Taipei 11529, Taiwan \\ ${ }^{3}$ Department of Physics, National Dong Hwa University, Hualien 97401, Taiwan
}

\begin{abstract}
We analytically study the photorefractive Gunn effect in n-GaAs subjected to two external laser beams which form a moving interference pattern (MIP) in the semiconductor. When the intensity of the spatially independent part of the MIP, denoted by $I_{0}$, is small, the system has a periodic domain train (PDT), consistent with the results of linear stability analysis. When $I_{0}$ is large, the space-charge field induced by the MIP will compete with the PDT and result in complex dynamics, including driven chaos via quasiperiodic route.
\end{abstract}

PACS number(s): 72.20.Ht, 42.70.Nq, 05.45.-a, 05.45.Yv

Keywords: A. Semiconductors; D. Pattern Competition; E. Nonlinear Analysis 
Spatiotemporal behavior of Gunn domains [1,2] in semiconductors is an interesting and important current research problem [3-8]. Gunn diodes are not only good systems for studying nonlinear dynamics, but also potentially important for various applications [7 9]. In 1996-1997 Segev et al. [3] and Subačius et al. [4] showed, respectively, that optical waves can excite multiple Gunn-domain formation with spatial periodicity, called periodic domain trains (PDT), in deep-impurity doped GaAs and semi-insulating GaAs; both groups used the well known Kroemer's criterion [1] to determine the number of Gunn domains. In 1998, Bonilla et al. [5] numerically studied the same dynamical system as Segev et al.; however, their findings are mostly inconsistent with the predictions of Segev et al.. Bonilla et al. [5] found that the number of Gunn domains in the system is not consistent with the prediction of Segev et al. and in many cases the system shows complex or chaotic behavior. Very recently, Shiau et al. used a linear stability analysis (LSA) to study a shallow-impurity doped GaAs with length $L$ subjected to two external laser beams which form a moving interference pattern (MIP) of intensity $I(x, t)=I_{0}[1+m \cos (K x+\Omega t)]$ and predicted PDT in the Gunn diode [7]. Here $\Omega$ is the frequency difference of the two optical waves, $K=2 \pi / \Lambda$ is the interference wave number, $\Lambda$ is the grating period and is much smaller than $L, m$ is the modulation depth, and $I_{0}$ is the average intensity. With consideration of time-delay feedback, the diode will emit high-dimensional chaotic microwaves, which is potentially useful for secure microwave communications, memory devices, applications involving photorefractive effects, etc. [7].

The purpose of this Communication is to clarify different findings of [3], [4], and [5] using a nonlinear analytic method to study the system of [7,8] as $L \rightarrow \infty$. In this approach, the intensity of the spatially independent part of the MIP, $I_{0}$, plays an important role in determining the simple dynamics (i.e., PDT) or complex dynamics of Gunn domains. When $I_{0}$ is small enough, the system shows simple dynamics consistent with the result of the LSA, and Kroemer's criterion is still valid. When $I_{0}$ is large, the space-charge field induced by the MIP will compete with the PDT and result in complex dynamics. This picture is also useful for understanding the results of other groups [3 5] discussed above. 
We consider two optical waves incident on a $n$-GaAs with a biased voltage $V$. The photon energy is just above the bandgap of GaAs, i.e., $1.42 \mathrm{eV}$, so that electron-hole pairs can be generated by optical excitation. The generation-recombination processes include complete thermal ionization of the donor, generation of electron-hole pairs by the MIP with rate $g$, and recombination of electron-hole pairs with rate $\gamma$. Therefore we can write the dynamical equations for the electric field $E$, the free electron density $n$, and the free hole density $p$ as:

$$
\begin{gathered}
\frac{\partial E}{\partial x}=\frac{e}{\epsilon}\left(n-N_{D}^{*}-p\right), \\
\frac{\partial n}{\partial t}=g I(x, t)-\gamma n p-\frac{\partial}{\partial x}\left[n v(E)-D_{n} \frac{\partial n}{\partial x}\right], \\
\frac{\partial p}{\partial t}=g I(x, t)-\gamma n p+\frac{\partial}{\partial x}\left[p \mu_{p} E+D_{p} \frac{\partial p}{\partial x}\right], \\
V=\int_{0}^{L} E(x, t) d x .
\end{gathered}
$$

Eq. (11) is for the Gauss law. Eq. (2) and Eq. (3) represent the continuity equations for the electrons and holes, respectively. The circuit condition is given at Eq. (4). Here $v(E)$ displays an N-shaped negative differential mobility (NDM). The homogeneous solution of Eqs. (回)-(四) is $E_{0}=V / L, n_{0}=\left(N_{D}^{*}+\sqrt{N_{D}^{* 2}+4 g I_{0} / \gamma}\right) / 2$, and $p_{0}=\left(-N_{D}^{*}+\sqrt{N_{D}^{* 2}+4 g I_{0} / \gamma}\right) / 2$. When $I_{0}$ is very small, $n_{0} \approx N_{D}^{*}+g I_{0} / N_{D}^{*} \gamma, p_{0} \approx g I_{0} / N_{D}^{*} \gamma$, and a natural expansion parameter for high order corrections is $\bar{\epsilon}=p_{0} / N_{D}^{*}=g I_{0} / N_{D}^{* 2} \gamma$. We can rearrange the model equations by the following rescaling process for the dynamical variables and parameters in Eqs. (四)-(田): $E \rightarrow E, \quad n \rightarrow n, \quad p \rightarrow \bar{\epsilon} p, \quad I \rightarrow \bar{\epsilon} I$. The small parameter $\bar{\epsilon}$ can distinguish the large variables (i.e., $E$ and $n$ ) from small variable $p$ in our model. Rescaling variables in Eqs. (苗)-(函), we have 《8] $\partial E / \partial x=e\left(n-N_{D}^{*}\right) / \epsilon+O(\bar{\epsilon}), \partial n / \partial t=-\partial\left[n v(E)-D_{n} \partial n / \partial x\right] / \partial x+O(\bar{\epsilon})$. These results and Eq. (3) implies

$$
\begin{gathered}
\frac{\partial E}{\partial t}=-\frac{1}{\epsilon} e N_{D}^{*} v(E)-v(E) \frac{\partial E}{\partial x}+D_{n} \frac{\partial^{2} E}{\partial x^{2}}+\frac{1}{\epsilon} J_{t o t}(t), \\
\frac{\partial p}{\partial t}=g I(x, t)-\gamma\left(N_{D}^{*}+\frac{\epsilon}{e} \frac{\partial E}{\partial x}\right) p+\frac{\partial}{\partial x}\left(p \mu_{p} E+D_{p} \frac{\partial p}{\partial x}\right),
\end{gathered}
$$


where $J_{t o t}(t)$ is time-dependent current density. The physical meaning of Eq. (5) is quite clear, which represents the Gunn-domain formation and with drift velocity $v\left(E_{0}\right)$ [1]. Then, Eq. (5) confirms our LSA results in Ref. [7]. In the following we want to show the detailed structure of PDT.

In order to get the solution of Eq. (5), an ansatz of the electric field, we set $E(x, t)=E_{0}+\left[E_{s}(x, t) e^{i \bar{K}\left(x-v_{0} t\right)}+E_{s}^{*}(x, t) e^{-i \bar{K}\left(x-v_{0} t\right)}\right] / 2$, where $v_{0}=v\left(E_{0}\right) . \quad E_{s}(x, t)$ is a new variable corresponding to the amplitude of electric-field domains and $E_{s}^{*}(x, t)$ is its complex conjugate; $\bar{K}(=2 \pi / L)$ is a bulk property of semiconductor. Since $\bar{K}$ is small, the spatiotemporal behavior of the dynamical system within the linear dimension $\Lambda$ is dominated by the behavior of $E_{s}(x, t)$. Actually, $\bar{K}$ is an irrelevent parameter which is verified by the numerical works of $[4]$. When $E_{0} \gg\left|E_{s}\right|$, we expand $v(E)$ as: $v(E) \approx v_{0}+v_{0}^{(1)}\left(E-E_{0}\right)+v_{0}^{(2)}\left(E-E_{0}\right)^{2} / 2+v_{0}^{(3)}\left(E-E_{0}\right)^{3} / 6$, where $v_{0}^{(j)}=d^{j} v(E) /\left.d E^{j}\right|_{E=E_{0}}$. Equation (5) and above expansions for $E(x, t)$ and $v(E)$ imply

$$
\begin{aligned}
\frac{\partial E_{s}}{\partial t}= & \left(-\frac{e}{\epsilon} N_{D}^{*} v_{0}^{(1)}-D_{n} \bar{K}^{2}\right) E_{s}+\left(i 2 D_{n} \bar{K}-v_{0}\right) \frac{\partial E_{s}}{\partial x}-\frac{1}{8} v_{0}^{(2)}\left(E_{s}^{2} \frac{\partial E_{s}^{*}}{\partial x}+2\left|E_{s}\right|^{2} \frac{\partial E_{s}}{\partial x}\right) \\
& +D_{n} \frac{\partial^{2} E_{s}}{\partial x^{2}}-\left(\frac{1}{8} \frac{e}{\epsilon} N_{D}^{*} v_{0}^{(3)}+i \frac{1}{8} v_{0}^{(2)} \bar{K}\right)\left|E_{s}\right|^{2} E_{s} .
\end{aligned}
$$

With $\bar{K} \rightarrow 0$ (as length $L \rightarrow \infty$ ) and $E_{s} \sim \Psi(t) e^{i K x}$, Eq. (7) implies

$$
\frac{d \Psi}{d t}=-C \Psi-\frac{1}{8}\left(\frac{e}{\epsilon} N_{D}^{*} v_{0}^{(3)}+i K v_{0}^{(2)}\right)|\Psi|^{2} \Psi
$$

Here $C=\left(e N_{D}^{*} v_{0}^{(1)} / \epsilon+D_{n} K^{2}+i K v_{0}\right)$. With $\Psi(t)=R(t) \exp i \Theta(t)$, Eq. (8) implies $R(t)=$ $-(a / b)^{1 / 2}\left[r_{0}^{2} e^{-2 a t} /\left(r_{0}^{2}\left(1-e^{-2 a t}\right)+a\right)\right]^{1 / 2}, \Theta(t)=-K v_{0} t-\frac{1}{8} K v_{0}^{(2)} \int^{t} R^{2}\left(t^{\prime}\right) d t^{\prime}$, where $a=$ $e N_{D}^{*} v_{0}^{(1)} / \epsilon+D_{n} K^{2}, b=e N_{D}^{*} v_{0}^{(3)} / 8 \epsilon$, and $r_{0}$ is related to initial value of $R(t)$. When $a>0$ (i.e., the operating point is within the regime of positive differential mobility PDM), $b>0$, and $t \rightarrow \infty, R(t)$ and $\Theta(t)$ tend to 0 and $-K v_{0} t$, respectively. These results mean that the origin on the complex plane of $\Psi(t)$ is a stable focus, which also implies electric-field profile in material is homogeneous, i.e., $E(x, t)=E_{0}$. When $a<0$ (i.e., the NDM regime), $b>0$, and $t \rightarrow \infty, R(t)=(-a / b)^{1 / 2}$ and $\Theta(t)=-K\left(v_{0}+a v_{0}^{(2)} / 8 b\right) t$. This solution describes a circular trajectory with counterclockwise angular frequency $K\left(v_{0}+a v_{0}^{(2)} / 8 b\right)$, radius $(-a / b)^{1 / 2}$, and 
transient response time $|a|^{-1}$. The transition from stable focus to stable limit cycle is a supercritical Hopf bifurcation. Since $\bar{K} \rightarrow 0, E_{s} \sim \Psi(t) e^{i K x}$ and $\Psi(t)=R(t) \exp i \Theta(t)$ imply for $a<0, b>0$, and $t \rightarrow \infty$ :

$$
E(x, t)=E_{0}+(-a / b)^{1 / 2} \cos \left[K\left(x-v_{0} t-a v_{0}^{(2)} t / 8 b\right)\right]
$$

which means that the PDT has a spatial period $\Lambda$ and a traveling velocity $v_{0}+a v_{0}^{(2)} / 8 b$. Since $v_{0}\left(\sim 10^{7} \mathrm{~cm} / \mathrm{s}\right)$ is much larger than $\left|a v_{0}^{(2)} / 8 b\right|$, so we neglect the term $a v_{0}^{(2)} / 8 b$ below.

In general, $E_{s}$ may be written as

$$
E_{s}=\sum_{q=1}^{\infty} \Psi_{q}(t) e^{i q K x}=\sum_{q=1}^{\infty}-\left(\frac{a_{q}}{b}\right)^{1 / 2}\left[\frac{r_{0}^{2} e^{-2 a_{q} t}}{r_{0}^{2}\left(1-e^{-2 a_{q} t}\right)+a_{q}}\right]^{1 / 2} e^{i q K\left(x-v_{0} t\right)} .
$$

where $a_{q}=e N_{D}^{*} v_{0}^{(1)} / \epsilon+D_{n} K^{2} q^{2}$. However, Eq. (10) needs to be corrected when we consider the coupling between spatial modes. This is very important for self-organizing systems. For example, consider two spatial modes $\Psi_{1} e^{i k x}$ and $\Psi_{2} e^{i 2 k x}$ with $a_{2}>0>a_{1}$ and $b>0$. From equations for $R(t)$ and $\Theta(t)$, we know that $\left|\Psi_{1}\right| \rightarrow\left(-a_{1} / b\right)^{1 / 2}$ and $\left|\Psi_{2}\right| \rightarrow 0$ when $t \rightarrow \infty$. Then $\left|\Psi_{2}\right| /\left|\Psi_{1}\right|$ will become zero. This result implies that only unstable spatial modes (i.e., $\left.a_{q}<0\right)$ have contributions for pattern-forming systems. However, if we combine these two modes, i.e., $E(x, t)=E_{0}+\sum_{q=1}^{2}\left[\Psi_{q}(t) e^{i q K x}+\Psi_{q}^{*}(t) e^{-i q K x}\right] / 2$, and substitute it into Eq. (5), we have

$$
\begin{aligned}
& \frac{d \Psi_{1}}{d t}=-\left(a_{1}+i K v_{0}\right) \Psi_{1}-\sum_{m_{1}+m_{2}+\cdots+m_{s}=1} \frac{\beta_{s, 1}}{s !} \Psi_{m_{1}} \Psi_{m_{2}} \cdots \Psi_{m_{s}}, \\
& \frac{d \Psi_{2}}{d t}=-\left(a_{2}+i 2 K v_{0}\right) \Psi_{2}-\sum_{m_{1}+m_{2}+\cdots+m_{s}=2} \frac{\beta_{s, 2}}{s !} \Psi_{m_{1}} \Psi_{m_{2}} \cdots \Psi_{m_{s}}
\end{aligned}
$$

where $m_{s}= \pm 1$ or $\pm 2, \beta_{s, m}=e N_{D}^{*} v_{0}^{(s)} / \epsilon+i m s v_{0}^{(s-1)} K$, and $\Psi_{q}^{*}=\Psi_{-q}$ [6]. Based on the slaving principle [10], we know that the stable mode $\Psi_{2}$ will be determined by the unstable mode $\Psi_{1}$, and that $\Psi_{2}$, and hence the ratio $\left|\Psi_{2}\right| /\left|\Psi_{1}\right| \equiv r_{2,1}\left(K, N_{D}^{*}\right)$, will be nonzero. For simplicity, in the following we assume that $r_{q+1, q}\left(\equiv\left|\Psi_{q+1}\right| /\left|\Psi_{q}\right|\right)$ is independent of $q$ and denote the number by $r$, which can be determined by experiments (see below). From $E(x, t)=E_{0}+\left[E_{s}(x, t)+E_{s}^{*}(x, t)\right] / 2$ and Eq. (10), for large $t$ we get 


$$
E(x, t)=E_{0}+\left(\frac{-a_{1}}{b}\right)^{1 / 2} \frac{-r+\cos \left[K\left(x-v_{0} t\right)\right]}{1+r^{2}-2 r \cos \left[K\left(x-v_{0} t\right)\right]}
$$

Therefore, the maximum and minimum fields are equal to $E_{\max }=E_{0}+\left(-a_{1} / b\right)^{1 / 2}(1-$ $r)^{-1}$ and $E_{\min }=E_{0}-\left(-a_{1} / b\right)^{1 / 2}(1+r)^{-1}$, respectively. According to Pockel's effect, the difference in refractive index between $E_{\max }$ and $E_{\min }$ is $-\bar{n}^{3} \gamma_{41}\left(-a_{1} / b\right)^{1 / 2}\left(1-r^{2}\right)^{-1}$, where $\bar{n}$ is refractive index of pure material and $\gamma_{41}$ is the electro-optic coefficient $(\bar{n}=3.3$ and $\gamma_{41}=1.43 \times 10^{-10} \mathrm{~cm} / \mathrm{V}$ for GaAs when the wavelength of the incident light is $\left.1 \mu \mathrm{m}\right)$. The profile of Eq. (13), shown in Fig. 1, is strongly $r$ dependent. If $r$ is near $0, E(x, t)$ is approxmately equal to $\cos \left[K\left(x-v_{0} t\right)\right]$. If $r$ is near 1 , Eq. (13) shows an obvious shockwave structure. In some senses, $r$ is related to the product $N_{D}^{*} \Lambda$ in Kroemer's criterion for domain formation. To see this, we first consider $r \rightarrow 1$. In this case, $\left(\left|\Psi_{q}\right|-\left|\Psi_{q+1}\right|\right) /\left|\Psi_{q}\right|$, and hence $\left(\left|a_{2}\right|-\left|a_{1}\right|\right) /\left|a_{1}\right|$, is much smaller than 1 and there are many excited unstable Fourier modes. Thus, $-e N_{D}^{*} v_{0}^{(1)} / \epsilon$ will be much larger than $D_{n} K^{2}$ and it follows from Eq. (10) that Kroemer's criterion for sharp domain formation is $a_{1} \Lambda / v_{0} \approx e N_{D}^{*} v_{0}^{(1)} \Lambda /\left(v_{0} \epsilon\right) \gg 1$, i.e. $N_{D}^{*} \Lambda \gg-v_{0} \epsilon e^{-1} / v_{0}^{(1)}\left(\sim 10^{12} \mathrm{~cm}^{-2}\right)$. On the other hand, in the case $r \rightarrow 0,-e N_{D}^{*} v_{0}^{(1)} / \epsilon$ is of the same order as $D_{n} K^{2}$ and Kroemer's criterion for the appearance of sinusoidal electricfield domain is $a_{1} \Lambda / v_{0} \approx e N_{D}^{*} v_{0}^{(1)} \Lambda /\left(v_{0} \epsilon\right) \approx 1$, i.e. $N_{D}^{*} \Lambda \approx-v_{0} \epsilon e^{-1} / v_{0}^{(1)}\left(\sim 10^{12} \mathrm{~cm}^{-2}\right)$. Therefore, Kroemer's criterion appears in Eq. (13) via the representation of $r$ parameter. To determine the relation between $r$ and $N_{D}^{*} \Lambda$, we can measure $\left(E_{\max }-E_{0}\right) /\left(E_{0}-E_{\min }\right)$ $(=(1+r) /(1-r))$ at different values of $N_{D}^{*} \Lambda$. Then we will find that $r$ is a function of $N_{D}^{*} \Lambda$. In other words, $r$ can be determined by experiments.

In order to get hole distribution, we assume $p(x, t)=p_{0}+\left[p_{s}(x, t) e^{i \bar{K}\left(x+\mu_{p} E_{0} t\right)}+\right.$ $\left.p_{s}^{*}(x, t) e^{-i \bar{K}\left(x+\mu_{p} E_{0} t\right)}\right] / 2$, and substitute such $p(x, t)$ into Eq. (6) to find

$$
\frac{\partial p_{s}}{\partial t}=-\left(D_{p} \bar{K}^{2}+\gamma N_{D}^{*}\right) p_{s}+D_{p} \frac{\partial^{2} p_{s}}{\partial x^{2}}+\left(2 i \bar{K} D_{p}+\mu_{p} E_{0}\right) \frac{\partial p_{s}}{\partial x}+S(x, t)
$$

Here $S(x, t)=2 g I_{0} e^{-i \bar{K}\left(x+\mu_{p} E_{0} t\right)} m \cos (K x+\Omega t)+p_{0}\left(\mu_{p}-\gamma \epsilon / e\right)\left(\frac{\partial E_{s}}{\partial x}+i \bar{K} E_{s}\right) e^{-i \bar{K}\left(v_{0}+\mu_{p} E_{0}\right) t}$. Equation (14) is a linear partial differential equation with external driving function $S(x, t)$ including the MIP and multiple Gunn domains. Thus $p_{s}$ can be considered as a linear 
combination of a homogeneous solution $p_{s}^{h}(x, t)$ and an inhomogeneous solution $p_{s}^{i}(x, t)$. Since the characteristic length of $p_{s}$ is equal to $\Lambda(\ll L)$, we may treat $\bar{K}$ as zero in Eq. (14) and write $\partial p_{s}^{h} / \partial t=-\gamma N_{D}^{*} p_{s}^{h}+D_{p} \partial^{2} p_{s}^{h} / \partial x^{2}+\mu_{p} E_{0} \partial p_{s}^{h} / \partial x$,

$$
\frac{\partial p_{s}^{i}}{\partial t}=-\gamma N_{D}^{*} p_{s}^{i}+D_{p} \frac{\partial^{2} p_{s}^{i}}{\partial x^{2}}+\mu_{p} E_{0} \frac{\partial p_{s}^{i}}{\partial x}+2 g I_{0} m \cos (K x+\Omega t)+p_{0}\left(\mu_{p}-\gamma \frac{\epsilon}{e}\right) \frac{\partial E_{s}}{\partial x} .
$$

It is easy to find solution of $p_{s}^{h}$ and show that $p_{s}^{h}=0$ as $t \rightarrow \infty$. When $E_{s}=$ $\sum_{q=1}^{\infty} r^{q-1}\left(-a_{1} / b\right)^{1 / 2} \exp \left[i q K\left(x-v_{0} t\right)\right]$ is substituted into Eq. (15), we can find solution for $p_{s}^{i}$. Therefore, the hole distribution $p(x, t)=p_{0}+\operatorname{Re}\left(p_{s}^{i}\right)$ is

$$
\begin{aligned}
& p(x, t)=\frac{g I_{0}}{\gamma N_{D}^{*}}+\frac{2 g I_{0} m}{A}\left[\left(D_{p} K^{2}+\gamma N_{D}^{*}\right) \sin (K x+\Omega t)-\left(K \mu_{p} E_{0}-\Omega\right) \cos (K x+\Omega t)\right] \\
& -D \sum_{q=1}^{\infty} \frac{q r^{q-1}\left\{q\left(v_{0}+\mu_{p} E_{0}\right) \cos \left[q K\left(x-v_{0} t\right)\right]+\left(\gamma N_{D}^{*}+q^{2} K^{2} D_{p}\right) \sin \left[q K\left(x-v_{0} t\right)\right]\right\}}{\left(\gamma N_{D}^{*}+q^{2} K^{2} D_{p}\right)^{2}+q^{2} K^{2}\left(v_{0}+\mu_{p} E_{0}\right)^{2}} .
\end{aligned}
$$

Here $A=\left(D_{p} K^{2}+\gamma N_{D}^{*}\right)^{2}+\left(K \mu_{p} E_{0}-\Omega\right)^{2}$ and $D=g I_{0} K\left(\mu_{p}-\gamma \epsilon / e\right)\left(-a_{1} / b\right)^{1 / 2} / \gamma N_{D}^{*}$. The pattern profiles of Eqs. (13) and (16) correspond to simple dynamics.

When $I_{0}$ is large and the higher order corrections in Eqs. (11)-(传) need to be included, then we have

$$
\begin{gathered}
\partial E / \partial x-e\left(n-N_{D}^{*}\right) / \epsilon=-\bar{\epsilon} e / \epsilon p^{(0)} \\
\frac{\partial n}{\partial t}+\frac{\partial}{\partial x}\left[n v(E)-D_{n} \frac{\partial n}{\partial x}\right]=\bar{\epsilon}\left[g I(x, t)-\gamma\left(N_{D}^{*}+\frac{\epsilon}{e} \frac{\partial E^{(0)}}{\partial x}\right) p^{(0)}\right],
\end{gathered}
$$

where $p^{(0)}$ and $E^{(0)}$ are solutions of Eqs. (16) and (13), respectively. These results imply that up to order $O(\bar{\epsilon})$ we have

$$
\frac{\partial E}{\partial t}+\frac{1}{\epsilon} e N_{D}^{*} v(E)+v(E) \frac{\partial E}{\partial x}-D_{n} \frac{\partial^{2} E}{\partial x^{2}}-\frac{1}{\epsilon} J_{t o t}(t)=\bar{S}(x, t),
$$

where $\bar{S}(x, t)=\bar{\epsilon} e\left[p^{(0)} v\left(E^{(0)}\right)-\mu_{p} E^{(0)} p^{(0)}-\left(D_{p}+D_{n}\right) \partial p^{(0)} / \partial x\right] / \epsilon$ and represents the electron-hole coupling. To understand the underlying physics of Eq. (19), we follow the same procedure as the case of simple dynamics, to get

$$
\frac{d \Psi}{d t}=-C \Psi-\frac{1}{8}\left(\frac{e}{\epsilon} N_{D}^{*} v_{0}^{(3)}+i K v_{0}^{(2)}\right)|\Psi|^{2} \Psi+\int_{0}^{\Lambda} \bar{S}(x, t) e^{-i K x} d x
$$


which is a generalization of Eq. (8) and represents a self-sustained oscillator (i.e., PDT) under the external driving force $\bar{\epsilon} F(t) / \epsilon\left(\equiv \int_{0}^{\Lambda} \bar{S}(x, t) e^{-i K x} d x\right)$ and

$$
\begin{aligned}
& F(t)=\left[i E_{0} \mu_{p} \frac{\pi}{K}-i v_{0} \frac{\pi}{K}-\left(D_{p}+D_{n}\right) \pi\right] G_{1} e^{i \Omega t}-\left[E_{0} \mu_{p} \frac{\pi}{K}-v_{0} \frac{\pi}{K}+i\left(D_{p}+D_{n}\right) \pi\right] G_{2} e^{i \Omega t} \\
& -\left[E_{0} \mu_{p} \frac{\pi}{K}-v_{0} \frac{\pi}{K}+i\left(D_{p}+D_{n}\right) \pi\right] G_{3} e^{-i K v_{0} t}+\left[i E_{0} \mu_{p} \frac{\pi}{K}-i v_{0} \frac{\pi}{K}-\left(D_{p}+D_{n}\right) \pi\right] G_{4} e^{-i K v_{0} t} \\
& +\frac{\pi}{K}\left(\frac{-a_{1}}{b}\right)^{1 / 2}\left(v_{0}^{(1)}-\frac{a_{1}}{8 b} v_{0}^{(3)}-\mu_{p}\right) G_{0} e^{-i K v_{0} t}
\end{aligned}
$$

where $G_{0}=g I_{0} / \gamma N_{D}^{*}, G_{1}=2 g I_{0} m\left(D_{p} K^{2}+\gamma N_{D}^{*}\right) / A, G_{2}=-2 g I_{0} m\left(K \mu_{p} E_{0}-\Omega\right) / A, G_{3}=$ $-g I_{0} K^{2}\left(\mu_{p}-\gamma \epsilon / e\right)\left(-a_{1} / b\right)^{1 / 2}\left(v_{0}+\mu_{p} E_{0}\right) / B, G_{4}=-g I_{0} K\left(\mu_{p}-\gamma \epsilon / e\right)\left(-a_{1} / b\right)^{1 / 2}\left(\gamma N_{D}^{*}+\right.$ $\left.K^{2} D_{p}\right) / B$, and $B=\gamma N_{D}^{*}\left(\gamma N_{D}^{*}+K^{2} D_{p}\right)^{2}+K^{2}\left(v_{0}+\mu_{p} E_{0}\right)^{2}$. The intrinsic oscillating frequency in Eq. (20) is $v_{0} / \Lambda$ and the external driving frequencies include $v_{0} / \Lambda$ and $\Omega / 2 \pi$. If the operating point is in the PDM regime (i.e., $a_{1}>0$ ), the last three terms in Eq. (21) will be zero. This means that there is no Gunn-domain formation in the semiconductor, but the external optical waves, i.e., the first two terms on the right-hand side of Eq. (21), will trigger a space-charge field $(\mathrm{SCF}) \sim e^{i(K x+\Omega t)}$, which is well known in photorefractive material [9]. On the other hand, if the operating point is in the NDM regime, in addition to the SCF, the PDT will be generated by the intrinsic electrical instability with the oscillating frequency $K v_{0}$. The relative strength of the SCF and PDT depends on the strength of spatially independent part of the MIP, i.e. $I_{0}$. When $I_{0}$ is small, SCF may be neglected and the behavior of the system is dominated by the PDT. When $I_{0}$ is large, the SCF will compete with the PDT and the system will show complex dynamics. For example, these two competing frequencies $\Omega / 2 \pi$ and $v_{0} / \Lambda$ can lead to quasiperiodic behavior.

The picture presented above is useful for understanding the results of other groups. The system numerically studied by Subačius et al. [4 may be represented by Eqs.(11)-(化) of this Communication with $N_{D}^{*}=0$ and $\Omega=0$. When the system is illuminated by a pulse of light interference field with intensity $I(x, t)=I_{0} f(t)[1+m \cos (K x)]$ and $m<<1$ ( $f(t)$ is the temporal shape of a laser pulse), the temporal part $I_{0} f(t)$ generates background electrons in the conduction band and the very small spatially dependent part of the light makes a small modulation on the background. The situation is similar to the system of 
the present manuscript with very small $I_{0}$. Fig. 1 in the present Communication is very similar as the numerical results of Subačius et al.. On the other hand, Segev et al. [3] and Bonilla et al. [5] considered deep-impurity doped GaAs under the externally optical waves, i.e., $I(x, t)=I_{0}[1+m \cos (K x+\Omega t)]$. In their case the modulation depth $m$ also plays a crucial role. For example, Segev et al. considered $m$ as a small parameter. Then the dynamical variables can be analytically expanded to Fourier series based on the form of $I(x, t)$. Therefore they can get multiple Gunn-domain order by order. Actually the physical idea of Segev et al. is the same as Subačius et al.. The spatial period of $I(x, t)$ determines the spacing of Gunn domains and $I_{0}\left(\right.$ or $\left.I_{0} f(t)\right)$ generates background electrons. However the work of Segev et al. didn't discuss the stability of multiple Gunn-domain, which will lead to the travelling domains with the same moving velocity (i.e., $\Omega / K$ ) of $I(x, t)$. Furthermore, according to the prediction of Segev et al. the moving velocity of electric-field profile in PDM or NDM regime has no any difference. This unsuitable prediction can be addressed and verified by Eq. (20). As for the numerical work of Bonilla et al., they showed complicated spatiotemporal behaviors of Gunn domains, e.g., quasiperiodic route to chaos, are mostly observed in their simulations. In other words, PDT didn`t appear in their system. Therefore they concluded that Kroemer's criterion can not give the correct numbers of Gunn domains. We think that $m=0.1$ used in their study is large enough to generate driven chaos due to the competition between SCF and PDT. Therefore both considerations of Kroemer's criterion and pattern competition may give correct physics in light-triggered Gunn-domain systems.

In conclusion, using a nonlinear analytic method we confirm the LSA results of [7] and find that the competition between the SCF and the PDT could be a dominant factor to determine the spatiotemporal pattern in semiconductor. We hope that the formulation of the present Communication can be further verified by numerical calculations or experiments.

We thank Jonathan Dushoff for a critical reading of the paper. This work was supported in part by the National Science Council of the Republic of China (Taiwan) under Contract No. NSC 89-2112-M-001-084. 


\section{REFERENCES}

* Corresponding Author, Fax: 886-3-573-5086, Electronic address: yhshiau@phys.cts.nthu.edu.tw.

+ Electronic address: huck@phys.sinica.edu.tw.

[1] B. K. Ridley, Proc. Phys. Soc. Lond. 82, 954 (1963).

[2] J. B. Gunn, Solid State Commun. 1, 88 (1963).

[3] M. Segev, et al., Phys. Rev. Lett. 76, 3798 (1996) and 82, 1799 (1999).

[4] L. Subačius, V. Gružinskis, E. Starikov, P. Shiktorov, and K. Jarašiunas, Phys. Stat. Sol. (b) 204, 473 (1997).

[5] L. L. Bonilla, M. Kindelan, and P. J. Hernando, Phys. Rev. B 58, 7046 (1998).

[6] Y.-H. Shiau, et al., Phys. Rev. B 56, 9247 (1997); J. Phys. Soc. Jpn 67, 1050 (1998); Phys. Rev. E 57, R1227 (1998).

[7] Y.-H. Shiau, H.-P. Chiang, Y.-C. Cheng, and C.-K. Hu, preprint.

[8] Y.-H. Shiau, Phys. Rev. B 60, 15534 (1999).

[9] P. Yeh, Introduction to Photorefractive Nonlinear Optics, (John Wiley \& Sons, Inc., 1993).

[10] H. Haken, Synergetics, 3rd ed. (Springer, Berlin, Heidelberg, 1983). 


\section{Figure Caption}

FIG. 1. The profile of $E(x, t)$ represented by Eq. (13) for several values of $r$, where $E_{0}=10$ $\mathrm{kV} / \mathrm{cm},\left(-a_{1} / b\right)^{1 / 2}=9.8 \mathrm{kV} / \mathrm{cm}, \Lambda=15 \mu \mathrm{m}$, and $L=45 \mu \mathrm{m}$. 
Fig. 1 Shiau and $\mathrm{Hu}$

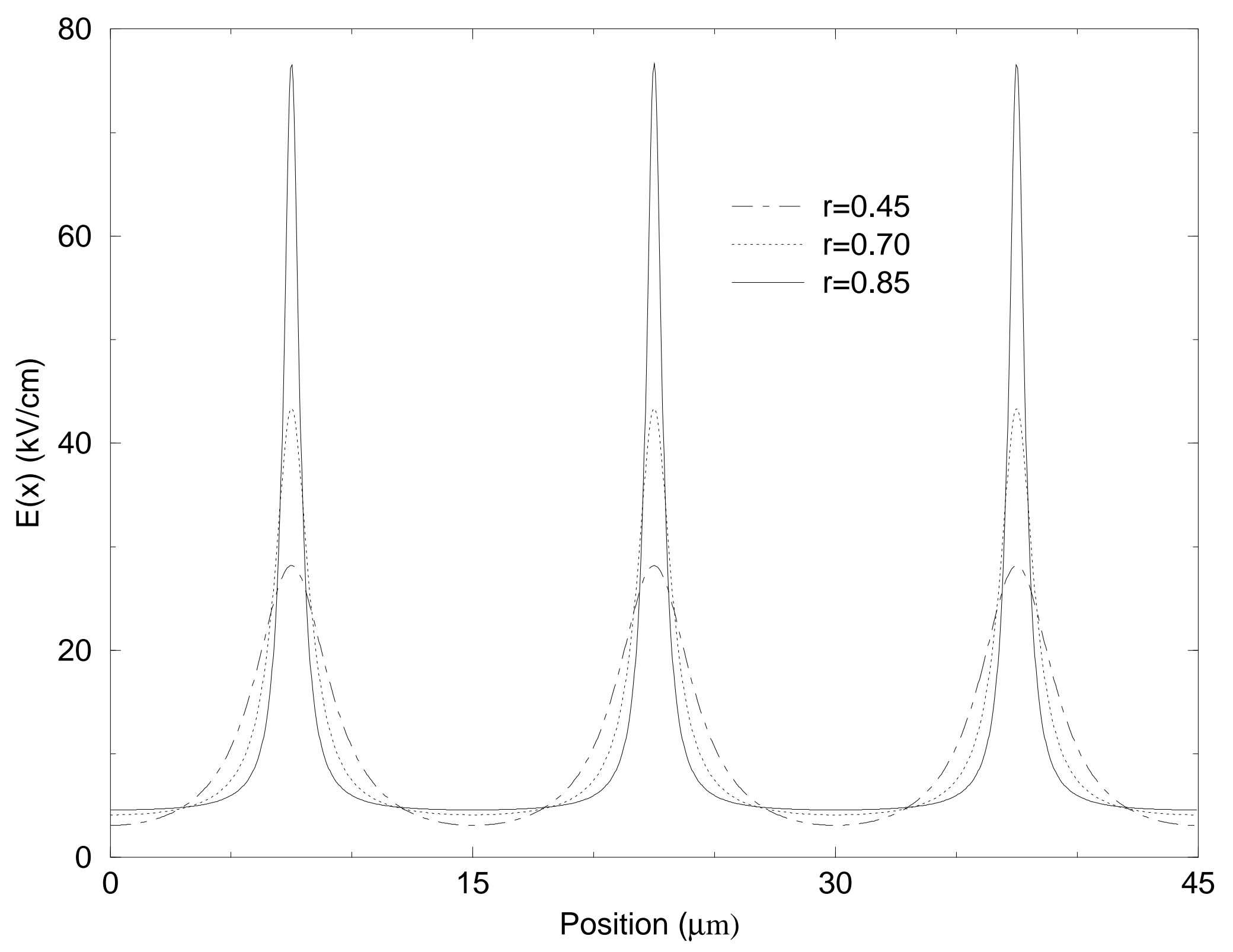

